\title{
Estilo de vida do homem e as doenças cardiovasculares: uma estratégia de promoção
}

\section{à saúde}

Men's lifestyle and cardiovascular diseases: a health promotion strategy

Estilo de vida de los hombres y enfermedades cardiovasculares: una estrategia de promoción de la salud

Karoline Lacerda de Oliveira

ORCID: https://orcid.org/0000-0001-8755-5858 Universidade do Estado do Rio de Janeiro, Brasil E-mail: lacerdakarol@hotmail.com

Renata Lopes Macedo

ORCID: https://orcid.org/0000-0001-7425-030X Universidade do Estado do Rio de Janeiro, Brasil

E-mail: renatalopesmacedo@gmail.com

Elizabeth Rose Costa Martins

ORCID: https://orcid.org/0000-0001-5947-5535 Universidade do Estado do Rio de Janeiro, Brasil E-mail: oigresrose@gmail.com

Andressa da Silva Medeiros ORCID: https://orcid.org/0000-0001-8677-2543 Universidade do Estado do Rio de Janeiro, Brasil E-mail: andressamedeirosmedeiros2@gmail.com

Letícia Guimarães Fassarella ORCID: https://orcid.org/0000-0002-3903-7383 Universidade do Estado do Rio de Janeiro, Brasil E-mail: lelefassarella@gmail.com

Fabiana Cristina Silva da Rocha ORCID: https://orcid.org/0000-0001-7665-0550 Universidade do Estado do Rio de Janeiro, Brasil E-mail: fabianacsrocha@hotmail.com

\begin{abstract}
Resumo
Objetivos: Descrever o estilo de vida do homem com doença cardiovascular; identificar a percepção do homem quanto a sua doença e suas formas de prevenção; discutir a promoção à saúde do homem e prevenção de agravos voltados às doenças cardiovasculares. Metodologia: Estudo descritivo e abordagem qualitativa, com 22 homens na faixa etária de 25 a 59 anos, com algum tipo de comorbidade relacionada às doenças cardiovasculares. Utilizou-se como instrumento de coleta de dados a entrevista semiestruturada e o método foi o de bola de neve, baseado em uma amostra não probabilística, em que um participante inicial indicou novos participantes. Para análise dos dados, foi aplicada a técnica de análise de conteúdo, conforme as seguintes etapas: pré-análise, exploração do material e tratamento dos resultados, inferência e interpretação. Optou-se por duas categorias: o homem cuidando de si e seu estilo de vida e a prevenção das doenças cardiovasculares na perspectiva do gênero masculino. Resultados e Discussão: Os resultados evidenciam que a maioria dos homens com doenças cardiovasculares possui um estilo de vida sedentário e uma visão do cuidado como feminino, trazendo como consequência agravos à saúde devido à falta de promoção e prevenção de agravos, reafirmando que a porta de entrada para cuidar da sua saúde é a emergência. Considerações Finais: É necessário investir em educação em saúde de modo a aproximar a atenção básica da população masculina, com utilização de uma linguagem acessível, além de capacitar profissionais para captar indivíduos desse grupo, ampliando, assim, o cuidado à saúde do homem.
\end{abstract}

Palavras-chave: Estilo de vida; Doença cardiovascular; Saúde do homem; Enfermagem.

\begin{abstract}
Objectives: to describe the lifestyle of men with cardiovascular disease; to evaluate men's perceptions of their disease and its prevention approaches; to discuss both men's health promotion and the prevention of aggravation regarding cardiovascular diseases. Methods: qualitative descriptive study on 22 men in the 25-59 age group presenting any comorbidities associated with cardiovascular diseases. Semi-structured interviewing was employed as the strategy for data collection with the snowball sampling method, a non-probability technique in which research participants recruited other participants. Regarding data analysis, content analysis method was used, a process divided into the following phases: pre-analysis, material exploration and treatment of results, inference and interpretation. Two
\end{abstract}


categories were selected: men's self-care and lifestyle, and the male perspective on the prevention of cardiovascular diseases. Results and Discussion: the data show that most of the men with cardiovascular diseases have a sedentary lifestyle and that they see self-care as a female concern, resulting in health issues due to the lack of health promotion and disease prevention, what reveals that emergency is the gateway to self-care. Final considerations: it is necessary to invest in health education to bring the basic health care closer to the male population reality using plain language, in addition to training professionals to recruit individuals from this group, thus expanding men's health care.

Keywords: Lifestyle; Cardiovascular disease; Men's health; Nursing.

\begin{abstract}
Resumen
Objetivos: Describir los estilos de vida del hombre con enfermedad cardiovascular; identificar la percepción del hombre en relación a su enfermedad y sus formas de prevención; discutir el fomento a la salud del hombre y prevención de agravios dirigidos a las enfermedades cardiovasculares. Metodología: Estudio descriptivo y enfoque cualitativo, con 22 hombres de franja etaria de 25 hasta 59 años, con algunos tipos de comorbilidad relacionada a las enfermedades cardiovasculares. Se utilizó como herramienta de recolección de datos las encuestas semiestructuradas y el método fue el de bola de nieve, basado en una muestra no probabilística, en el que un participante inicial indicó nuevos participantes. Para análisis de los datos, fue aplicada la técnica de análisis de contenido, según las siguientes fases: preanálisis de datos, exploración del material y tratamiento de los resultados, inferencia y interpretación. Se optó por dos categorías: el hombre cuidando a si mismo y su estilo de vida y la prevención de las enfermedades cardiovasculares en la perspectiva del género masculino. Resultado y debates: Los resultados evidencian que la mayoría de los hombres con enfermedades cardiovasculares poseen un estilo de vida sedentario y una visión de cuidado como algo femenino, trayendo como consecuencia agravios a la salud debido a la falta de fomento y prevención de agravios, reafirmando que el punto de ingreso para cuidar de su salud es la emergencia. Consideraciones finales: Hace necesario investir en educación en salud de manera a aproximar la atención básica de la población masculina, con la utilización de un lenguaje accesible, además de capacitar profesionales para captar sujetos del grupo, expandiendo, de este modo, el cuidado a la salud del hombre.
\end{abstract}

Palabras clave: Estilo de vida; Enfermedad cardiovascular; Salud de los hombres; Enfermería.

\title{
1. Introdução
}

Desde muito tempo, a saúde do homem tem sido pauta para sua inclusão no cenário dos serviços de saúde da atenção primária. Sendo assim, notou-se que os maiores influenciadores desse fenômeno são: a questão de gênero, que, muitas vezes, é fortemente carregada de preconceitos, e a questão da figura masculina na sociedade, contida nela o papel patriarcal e provedor da família. Nesse sentido, a presença masculina no cenário da saúde tornou-se um grande desafio para as políticas públicas e para os profissionais de saúde.

Na perspectiva de difundir a promoção e prevenção dentro do campo da saúde do homem, o Ministério da Saúde trouxe como prioridade, no ano de 2008, a Política Nacional de Atenção Integral à Saúde do Homem (PNAISH). Esta tem como um dos seus principais objetivos reconhecer a singularidade masculina dentro do contexto sociocultural, político e econômico, além de respeitar os níveis de organização dos sistemas de saúde, a fim de reduzir as taxas de morbimortalidade associadas às doenças preveníveis. Os agravos do sexo masculino constituem verdadeiros problemas de saúde pública; em relação à faixa etária escolhida, decidiu-se por participantes entre 25 e 59 anos de idade, uma vez que se entende que é a população masculina mais ativa e com mais necessidade de atenção (Brasil, 2008).

A proposição desta política visa qualificar a saúde da população masculina na perspectiva de linhas de cuidado que resguardem a integralidade e a qualidade da atenção, no entanto esses pontos dependem da adesão do grupo masculino aos tratamentos de saúde. Considerando os determinantes sociais da saúde, a PNAISH optou, como estratégia, pela identificação das principais enfermidades e agravos à saúde do homem, buscando delinear caminhos que incentivem este indivíduo a procurar pelos serviços de atenção primária antes que os agravos requeiram atenção especializada.

Segundo a PNAISH (Brasil, 2008), as doenças cardiovasculares (DCV) situam-se dentro dos cinco grupos que acarretam a mortalidade masculina, ocupando o segundo lugar após causas externas. As DCV, inseridas no grupo de doenças crônicas não transmissíveis, são responsáveis por aproximadamente $78 \%$ de óbitos em países de baixa e média renda, sendo a cardiopatia isquêmica alocada em primeiro lugar, seguida pelas doenças cerebrovasculares, as principais causas de morte no 
mundo. Segundo a Organização Pan-Americana da Saúde (OPAS, 2017), em 2016, cerca de 15,2 milhões de óbitos foram devido a esta morbidade.

De acordo com o último Censo Demográfico do Instituto Brasileiro de Geografia e Estatística (IBGE, 2018), atualmente existem no país aproximadamente 209,3 milhões de pessoas, sendo que 48,3\% formam a população masculina. Os homens vivem, em média, sete anos e meio a menos que as mulheres. As principais causas de mortalidade masculina entre $20 \mathrm{e}$ 59 anos são as causas externas, como agressões e acidentes de veículos, que correspondem a 89.528 óbitos (36,4\%). Em seguida, vêm as doenças do aparelho circulatório - infarto agudo do miocárdio, acidente vascular cerebral, insuficiência cardíaca -, que correspondem a 43.518 óbitos (17,7\%) entre outros (Inca, 2015).

Segundo Albuquerque et al. (2014), os homens são mais vulneráveis a doenças em comparação à mulher devido à maior exposição a fatores de risco comportamentais e culturais, além de ter que arcar com o estereótipo de gênero na sociedade que fortalece a desvalorização do autocuidado neste público. Outra condição agravante é o despreparo do enfermeiro para atender às particularidades destes indivíduos e a dificuldade desses profissionais em estimular a adesão dos homens à rede de atenção básica. Sendo assim, o homem, ao invés de buscar a prevenção, acaba adentrando no sistema de saúde através da atenção terciária, quando o agravo já se instalou, gerando um alto custo para o Sistema Único de Saúde (SUS) (Moreira, Fontes \& Barbosa, 2014).

O papel de não vulnerável leva o homem a se envolver em atividades muitas vezes agressivas e que põem em risco sua saúde. Além de se exporem mais a situações de risco, os homens têm mais resistência a procurarem os serviços de saúde. Esta resistência também se relaciona com a perspectiva de gênero e a invulnerabilidade masculina, o que implica diretamente nos índices epidemiológicos destes indivíduos (Silva, 2015).

Nesse contexto, definiu-se como objeto de estudo a relação entre estilo de vida e as doenças cardiovasculares na população masculina. Frente a isso, surgiram as seguintes questões norteadoras: Qual o estilo de vida do homem com doenças cardiovasculares? O homem se cuida, de modo a prevenir as doenças cardiovasculares? O homem faz exames preventivos?

Para dar conta das questões norteadoras, foi delimitado como objetivo geral compreender a relação do estilo de vida do homem com as doenças cardiovasculares. Como objetivos específicos foram definidos: Descrever o estilo de vida do homem com doença cardiovascular; Identificar a percepção do homem quanto a sua doença e suas formas de prevenção; Discutir a promoção à saúde do homem e a prevenção de agravos, ambas voltadas para as doenças cardiovasculares.

\section{Metodologia}

Trata-se de uma pesquisa exploratória, de natureza descritiva e abordagem qualitativa. A pesquisa qualitativa não se mostra restrita a um conjunto de variáveis ou resultados padronizados e fixos. Cada tema é explorado na sua individualidade de forma detalhada, buscando a compreensão do indivíduo acerca de um determinado fenômeno. A pesquisa de campo analisa dados diretamente da população escolhida, trazendo uma abordagem mais realística e próxima entre pesquisador-pesquisado. Com isso, o investigador apropria-se de conhecimentos que somente durante a vivência da realidade daquele grupo ele seria capaz de obter (Cyriacoa, Nunn, Amorim, Falcão \& Moreno, 2017).

Os participantes deste estudo foram homens com algum tipo de comorbidade relacionada às doenças cardiovasculares. O critério de inclusão foram homens acometidos de doenças cardiovasculares na faixa etária de 25 a 59 anos, uma vez que a política nacional de atenção integral à saúde do homem estabelece estes indivíduos como força produtiva do país, além de exercer um significativo papel sociocultural e político. Como critério de exclusão, ficaram definidos homens que não possuíam diagnóstico de doenças cardiovasculares (Brasil, 2008). O estudo contou com 22 participantes que estavam dentro do critério de inclusão e aceitaram participar da pesquisa. 
O estudo seguiu os aspectos éticos e as determinações contidas na Resolução 466/2012 do Conselho Nacional de Saúde (CNS/MS) que regulamenta pesquisas realizadas com seres humanos (Brasil, 2012). O projeto foi submetido ao Comitê de Ética e Pesquisa (CEP) de universidade pública no município do Rio de Janeiro e aprovado com o parecer nº 4.398.892.

Cada participante recebeu um termo de consentimento livre e esclarecido (TCLE) e foram orientados quanto aos objetivos do estudo, a relevância, a metodologia, assim como a garantia da participação voluntária, do sigilo e do anonimato, da possibilidade de desistência da participação na pesquisa e dos possíveis riscos mínimos a ela relacionados. Como forma de assistência aos participantes, mesmo sendo um estudo que oferece riscos mínimos, foram-lhes assegurados que, caso expressassem alguma necessidade desencadeada pela participação na pesquisa, poderiam ser atendidos pelo serviço psicossocial do hospital universitário da referida instituição.

Como instrumento de coleta de dados, foi utilizada a entrevista semiestruturada. Com o objetivo de manter o anonimato e sigilo do participante, as entrevistas foram identificadas com a letra $\mathrm{H}$, correspondente à palavra homem, e um número de acordo com a quantidade de entrevistas realizadas pelos pesquisadores (H: 01; H: 02; H: 03 até H: 22).

A entrevista foi composta de duas partes: a primeira, caracterizando os participantes segundo a faixa etária, estado civil, escolaridade e ocupação; a segunda, com questões abertas sobre a doenças cardiovasculares, tempo de doença e periodicidade de acompanhamento clínico.

Na coleta de dados, o método escolhido foi o de bola de neve, baseado em uma amostra não probabilística, na qual um participante inicial indica novos participantes que se enquadrem nos critérios de inclusão do estudo relacionado (Baldin \& Munhoz, 2011; Manzini, 2015).

A coleta de dados ocorreu no período de 21 de novembro de 2020 a 1 de fevereiro de 2021; o número de participantes inseridos no estudo foi apoiado no critério de saturação, cuja base é a percepção do pesquisador em relação à compreensão da lógica dos participantes, do grupo ou da coletividade estudada e a identificação de que esse conhecimento reflete a totalidade das múltiplas dimensões do objeto do estudo em questão (Minayo, 2013).

Após a coleta, os dados foram transcritos e organizados. Com a intenção de conhecer os significados por trás dos discursos emanados durante as entrevistas, o método utilizado foi o da análise de conteúdo, pois tudo que é dito é susceptível de ser submetido a este tipo de análise (Bardin, 2016).

O objeto da análise de conteúdo é a palavra, aspecto individual da linguagem; seu objetivo são os significados dos vocábulos, tentando compreender os atores e o ambiente onde estão inseridos; é organizada em três etapas: pré-análise; exploração do material e tratamento dos resultados; inferência e interpretação (Bardin, 2016).

Para a sistematização dos achados, realizou-se leitura flutuante, a qual fez emergir 159 unidades de registro, organizadas em duas categorias: O homem cuidando de si e o estilo de vida e A prevenção das doenças cardiovasculares na perspectiva do gênero masculino.

\section{Resultados e Discussão}

A fim de compreender os participantes do estudo, criou-se uma amostra estratificada de caracterização, abordando os indicadores sociais mínimos dos participantes e as informações relevantes ao estudo, como idade, estado civil, ocupação, escolaridade, doenças cardiovasculares, tempo de doença e periodicidade de acompanhamento clínico.

Em relação à faixa etária, destacam-se os homens com idade entre 46 e 59 anos, que são 15 (68\%), e entre 25 e 45 anos, 07 (32\%) participantes. Quanto ao estado civil, percebe-se uma predominância de homens casados, que moram com as suas companheiras - $15(68 \%)$-, $06(27 \%)$ solteiros e sem companheiras e $01(5 \%)$ viúvo. No que tange à ocupação dos entrevistados, identificam-se: 03 (14\%) professores; 03 (14\%) aposentados; 02 (9\%) administradores; 02 (9\%) autônomos; 01 
(4,5\%) engenheiro; 01 (4,5\%) enfermeiro; 01 (4,5\%) cuidador; 01 (4,5\%) advogado; 01 (4,5\%) propagandista; 01 (4,5\%) gerente; 01 (4,5\%) bancário; 01 (4,5\%) funcionário público; 02 (9\%) repositores; 01 (4,5\%) policial e 01 (4,5\%) motorista.

$\mathrm{Na}$ escolaridade, pode-se notar que todos os homens completaram o nível fundamental - $22(100 \%)$ - e que 18 (82\%) possuem nível médio, enquanto 08 (36\%) concluíram o nível superior, valorizando-se o possível conhecimento adquirido. Gomes, Nascimento e Araújo (2007) trazem, em seu estudo, que, independentemente do nível de escolaridade, os participantes apresentam o conceito de "ser homem" entendido como ideias opositoras ao que por eles é entendido como o "ser mulher". Os participantes que cursaram o ensino superior apresentaram um discurso mais elaborado e crítico, devido à maior propriedade intelectual, mas apesar de entenderem a importância do cuidado com a saúde, afirmaram que nem sempre agiam de acordo com esta percepção. Tais declarações apontam a cultura do gênero como um fator mais influenciador no cuidado da saúde, quando comparado à escolaridade.

Quanto às doenças cardiovasculares apresentadas pelos participantes, a que predomina é a hipertensão arterial - 14 (64\%). Esses dados corroboram com o estudo que avaliou o impacto econômico para o Sistema de Saúde de quatro comorbidades mais prevalentes, sendo relacionadas: insuficiência cardíaca, infarto do miocárdio, fibrilação atrial e hipertensão. Estas comorbidades são responsáveis por cerca de 46 milhões de pessoas doentes e custaram monetariamente 56 bilhões de reais aos cofres públicos em 2015, representando 5,5\% do total nacional da despesa com assistência de saúde (Stevens et al., 2018). Quanto ao tempo de doença cardiovascular apresentada pelo homem, 11 (50\%) disseram não saber informar e 07 (32\%) relataram ter menos de 10 anos. Esta situação retrata a atual realidade da saúde do homem e as barreiras socioculturais que se interpõem entre a população masculina, o cuidar de si e os serviços de saúde (Gomes, 2008).

A periodicidade de acompanhamento clínico dos homens mostra que 08 (36\%) vão à consulta duas vezes ao ano e 07 (32\%) uma vez ao ano, enquanto 03 (14\%) são acompanhados três vezes ao ano; outros 03 (14\%) frequentam as consultas somente de dois em dois anos. Apenas 01 (4,5\%) afirmou ter acompanhamento mensal.

\subsection{O homem cuidando de si e o estilo de vida}

Esta categoria compreende 98 unidades de registro e representa 62\% do corpus analisado. Descreve a percepção do homem sobre o cuidar de si e seus hábitos de vida, conforme as seguintes falas:

\section{Só procuro médico quando a situação agrava. (H.03)}

\section{Vou ao médico quando sinto algo grave. (H.01)}

Faz-se necessário observar que a pouca procura pelos serviços de atenção primária, entre os homens, se remete a hábitos de vida e de construção social que nos levam direto à narrativa de que o cuidar de si está mais associado às mulheres.

Martins et al. (2020a) realizaram um estudo com 150 homens, no qual 87\% dos participantes demonstraram recorrer ao serviço de saúde somente em momentos de urgência e emergência. Os fatores determinantes para tal comportamento são as questões que envolvem a masculinidade e as barreiras socioculturais que reafirmam e incentivam a falta de autocuidado para esta população. O descaso do homem com sua saúde repercute diretamente em seus índices epidemiológicos, gerando diagnósticos tardios e piores prognósticos na maioria dos casos.

Sobre o estilo de vida, os homens trazem as seguintes falas: 
Eu não faço nada. (H.10)

Eu tenho consciência que precisaria melhorar meu estilo de vida. (H.15)

Teixeira e Cruz (2016) discutem os fatores capazes de levar um indivíduo a um estilo de vida não saudável, sendo o mais apontado a questão do tempo, pois o trabalho os impossibilita de marcar consultas preventivas. Vale ressaltar que fatores de risco às doenças cardiovasculares são modificáveis, podendo ser corrigidos através da educação em saúde e mudanças no estilo de vida, mas para que isso aconteça, é necessário que o homem compreenda a necessidade de cuidar da sua saúde e, nesse sentido, modifique hábitos de vida.

Os participantes do estudo corroboram com esses achados, uma vez que suas falas trazem o trabalho como um fator impeditivo na busca por consultas preventivas, o que aumenta sua exposição a riscos e vulnerabilidade.

Eu sou atarefado, dividindo meu tempo entre trabalho e a família, o que torna lidar com a rotina moderadamente estressante, sem tempo para me cuidar. (H.08)

Gostaria de praticar exercícios físicos, mas não tenho tempo. (H.07)

De alguma forma, o homem percebe que não está se cuidando e conhece os possíveis fatores de risco às doenças cardiovasculares, como sedentarismo, alimentação inadequada dentre outros.

Sou sedentário, tenho sono desregulado e alimentação inadequada. Sei que isto tudo é ruim. (H.05)

Tenho alimentação baseada em gorduras e carboidratos. (H. 02)

Gostaria de praticar exercícios físicos, mas não tenho tempo. (H.07)

Pode-se observar que os participantes do estudo possuem dificuldade em se perceber e se assumir como vulneráveis, mesmo entendendo suas necessidades de saúde e fatores de risco. Martins et al. (2020b) reforçam esse pensamento ao discutir como esse problema de déficit da compreensão impacta diretamente na mudança de estilo de vida desse homem.

As falas mostram que alguns homens entendem que o cuidar de si se baseia no uso de medicamentos que controlem suas doenças e que essa medida já é o suficiente para a manutenção da sua saúde. Desse modo, tem-se a visão de que o homem não compreende a importância da promoção à saúde e que, de certa forma, espere adoecer para ser medicado.

Tomo remédio regularmente. $(\mathrm{H} .05)$

Uso de remédios para melhorar. (H. 21)

Tomando remédio vou melhorar. (H. 16)

O cuidar de si e a valorização do corpo no sentido da saúde não são questões colocadas na socialização dos homens. Para eles, um tratamento medicamentoso não precisa de um complemento comportamental como a implementação de hábitos mais saudáveis, por exemplo. Por isso, é importante criar estratégias que façam com que a população masculina procure os serviços de saúde de atenção primária, a fim de receber orientações quanto à importância de atividades físicas e de uma alimentação saudável, de modo a prevenir possíveis doenças cardiovasculares. 


\subsection{A prevenção das doenças cardiovasculares na perspectiva do gênero masculino}

Essa categoria representa $38 \%$ do corpus do estudo analisado. Aqui, são retratadas as ações desenvolvidas por homens que buscam a prevenção das doenças cardiovasculares. Percebe-se que alguns homens participantes do estudo buscam o atendimento como forma de prevenção a doenças, conforme as falas:

Eu fui ao médico para um check up, a pedido da minha mulher e descobri uma doença cardiovascular. (H. 02)

Faço exame de rotina há 20 anos, quando descobri uma DCV. (H.15)

Faço visita de rotina ao médico com minha mulher. (H. 20)

Ainda que com resistência, alguns homens procuram os serviços de saúde para o acompanhamento profissional por influência da companheira. Segundo Martins et al. (2020a), é comum que homens casados dependam de suas mulheres nos cuidados com a saúde. Desse modo, o casamento mostra-se, para esses homens, como um fator de proteção a riscos e agravos da saúde. Nos casos de homens mais novos, a influência da mãe ainda é muito presente para esta demanda. Poucos homens entendem genuinamente a importância da avaliação periódica de suas doenças, o que implica a perpetuação de possíveis agravos à saúde. Algumas falas trazem a não realização de ações preventivas, como:

Eu não faço nada. (H. 07)

Eu não tenho tempo. (H. 03)

Essas falas nos mostram que até a correria do dia a dia é usada como justificativa para o descuido com a saúde no que se refere à população masculina. No entanto, mulheres, mesmo com suas duplas jornadas, cujas tarefas domésticas se somam às de trabalho e repercutem de forma negativa sobre suas qualidades de vida, ainda assim são as pessoas que mais procuram os serviços de saúde, principalmente no que tange ao quesito promoção à saúde e prevenção de agravos (Zibetti \& Pereira, 2010).

Vale ressaltar que os homens, por não se cuidarem, e consequentemente não desenvolverem ações de prevenção, só descobrem a doença quando já está avançada.

Descobri a doença cardiovascular, buscando a emergência devido a um mal-estar! Peso no peito. (H. 17)

Descobri que tinha problemas após o primeiro infarto. (H.05)

Eu tinha muita dor na nuca, mas quando piorou busquei uma urgência. Aí veio o diagnóstico. (H.17)

Só procuro médico quando a situação agrava. (H 22)

Neste contexto, pode-se perceber nas falas que a ausência pela busca ao serviço voltado à promoção da saúde e prevenção de doenças denota que a consolidação do gênero masculino e os conceitos atribuídos e repassados ao longo dos anos ainda estão presentes em nossa sociedade. Estes indivíduos permanecem sem procurar e entender a busca pelos serviços de saúde como uma prática necessária para o cuidar de si (Pozzati et al., 2013).

A prevalência dos fatores de predisposição às DCV tem tomado proporções alarmantes e a baixa adesão da população masculina ao serviço primário de saúde tem se tornado uma realidade ainda na atualidade, e está diretamente ligada ao estigma social de invulnerabilidade, isto é, o indivíduo acredita que, por se homem, está imune a toda e qualquer doença. Esse fator 
coloca o homem como principal indivíduo no grupo de risco para o acometimento das DCV; o sobrepeso e a obesidade aumentaram nos últimos anos, o que significa presença de fatores de risco importantes para a ocorrência das doenças cardiovasculares (Stevens et al., 2018)

As medidas de promoção e prevenção realizadas na atenção primária vêm apresentando resultado na redução dos fatores de predisposição para o desenvolvimento das DCV. A mudança na rotina diária, envolvendo hábitos de vida saudáveis, traz grandes benefícios à saúde da população masculina.

A prática de exercício físico, associada a hábitos alimentares saudáveis, tem grande peso na redução do fator de predisposição às DCV como redução da pressão arterial, controle de peso corporal e níveis mais aceitáveis de colesterol no sangue.

Vale ressaltar a atuação do profissional enfermeiro como um dos membros mais atuantes na orientação das principais medidas que auxiliam na redução dos fatores de risco para a evolução das DCV.

\section{Considerações Finais}

Os resultados mostram que os homens com doenças cardiovasculares possuem, em sua maioria, um estilo de vida sedentário, em razão da intensa rotina de trabalho e compromissos, fazendo com que coloquem sua saúde em segundo plano. Além disso, foi possível confirmar que este perfil traz como consequência um agravo à saúde devido à falta de promoção e prevenção, ratificando que a porta de entrada destes usuários é a emergência.

Este adoecimento ocorre não só por causa das questões de gênero e masculinidade, que reafirmam que o cuidar é sempre feminino e, por isso, homens não têm necessidade de buscar acompanhamento para evitar danos. Há também o despreparo da rede de atenção básica no acesso e no acolhimento à população masculina. O fato de a Política Nacional de Atenção Integral à Saúde do Homem ser de 2008, o que a torna recente em comparação às outras, faz com que haja grande dificuldade em sua implementação nos serviços de saúde.

Com isso, os profissionais de saúde nem sempre estão preparados para atender às demandas devido ao desconhecimento das necessidades desta população, o que gera dificuldade em fornecer um cuidado integral e longitudinal. O enfermeiro como um dos principais agentes promotores de saúde, precisa ter conhecimento voltado para a população masculina, como também preparar sua equipe para que todos possam, através da educação em saúde, captar homens ainda saudáveis e trabalhar as diversas formas de se evitar a doença e suas complicações.

É preciso investir na educação em saúde para que a porta de entrada deste usuário seja a atenção básica e não mais a emergência, promovendo ações de saúde que contribuam para a compreensão da realidade singular masculina nos seus diversos contextos socioculturais e político-econômicos, como também incentivando a autonomia do sujeito ao fornecer conhecimento sobre sua situação no intuito de atender às necessidades dessa população, no que envolve a relação estilo de vida e as doenças cardiovasculares.

Os fatores de risco às doenças cardiovasculares são modificáveis, podendo ser corrigidos através da educação em saúde e mudanças no estilo de vida. Tais ações são um grande desafio para o gerenciamento do cuidado de enfermagem na perspectiva de gênero e oferecem importantes discussões sobre promoção e prevenção de agravos à saúde.

Desta forma, será possível fomentar a promoção à saúde e alterar prognósticos que envolvem modificações de estilo de vida, favorecendo a este grupo a compreensão de sua realidade e, quem sabe, uma perspectiva melhor de vida.

O estudo apresenta limitações ao retratar a realidade de 22 homens; desta forma, os resultados não podem ser generalizados, apesar de serem condizentes com demais investigações nacionais. Nesse sentido, faz-se necessária a compreensão da integralidade em saúde e dos homens na perspectiva relacional de gênero, tal como a vulnerabilidade 
masculina, entre outras questões, no intuito de atender às necessidades dessa população, no que envolve a relação estilo de vida e as doenças cardiovasculares.

A investigação pretende contribuir com a discussão acerca da temática "saúde do homem", que, hoje, faz parte da realidade social, tornando-se necessária a reflexão sobre a relação estilo de vida e as doenças cardiovasculares na população masculina. Através desta compreensão, será possível refletir sobre a saúde do homem, identificar lacunas e transformá-la para um cuidar de qualidade.

No que concerne ao ensino de enfermagem, busca-se destacar a saúde do homem para a formação de profissionais, num contexto no qual as relações podem ser estabelecidas a fim de se encontrarem significados fundamentais para compreender e propor ações pautadas em princípios do SUS e nas proposições da PNAISH.

Para os serviços, almeja-se que os resultados tragam subsídios para fomentar e fortalecer os profissionais de enfermagem na promoção à saúde e prevenção de doenças cardiovasculares à população masculina, principalmente na atenção básica de saúde.

Espera-se que os resultados da investigação contribuam para agregar conhecimentos sobre a relação entre estilo de vida e as doenças cardiovasculares na população masculina e que traga subsídios para fomentar e incentivar novas pesquisas sobre a temática, bem como estimular a discussão sobre gênero e suas implicações para o cuidado, abrindo, assim, caminhos para novos estudos.

O estudo contribui e incentiva novas pesquisas sobre a temática, bem como amplia o debate sobre gênero e suas implicações para o cuidado, principalmente quanto à saúde do homem, abrindo, assim, caminhos para a compreensão da evolução e aumento da visibilidade desta população.

\section{Referências}

Albuquerque, G. A. et al. (2014). O homem na atenção básica: percepções de enfermeiros sobre as implicações do gênero na saúde. Esc. Anna Nery, 18(4), 615-621. https://doi.org/10.5935/1414-8145.20140086.

Baldin, N., \& Munhoz, E. M. B. (2011). Snowball (bola de neve): uma técnica metodológica para pesquisa em educação ambiental comunitária. Anais do Congresso Nacional de Educação - Educere, Curitiba, PR, Brasil, 10.

Bardin, L. (2016). Análise de conteúdo. Tradução Luís Antero Reto, Augusto Pinheiro. Edições 70.

Brasil. Ministério da Saúde. (2008). Política Nacional de Atenção Integral à Saúde do Homem: Plano de Ação Nacional. http://portalsaude.saude.gov.br/i ages/pdf/2014/maio/21/CNSH-DOC-Plano-Nacional.PNAISH-2009-2011.pdf.

Brasil. Ministério da Saúde. (2012). Resolução $n^{\circ} 466$, de 12 de dezembro de 2012. Incorpora, sob a ótica do indivíduo e das coletividades, referenciais da bioética, tais como autonomia, não maleficência, beneficência, justiça e equidade, dentre outros, e visa a assegurar os direitos e deveres que dizem respeito aos participantes da pesquisa, à comunidade científica e ao Estado. Publicada no DOU no 12 . Seção 1 - Página 59. https://conselho.saude.gov.br/resolu coes/2012/Reso466.pdf

Cyriacoa, A. F. F., Nunn D., Amorim, R. F. B., Falcão, D. P., \& Moreno, H. (2017). Qualitative research: key concepts and a brief overview of its application in geriatrics/gerontology. Geriatr Gerontol Aging, 11(1), 4-9. 10.5327/Z2447-211520171700011

Gomes, R. (2008). Sexualidade masculina, gênero e saúde. Fiocruz.

Gomes, R., Nascimento, E.F., \& Araujo, F.C. (2007). Why do men use health services less than women? Explanations by men with low versus higher education. Cad. Saude Pública, 23(3), 565-574. https://DOI.Org./10.1590/S0102-311X2007000300015

IBGE. (2018). Projeções da população do Brasil e Unidades da Federação por sexo e idade: 2010-2060. https://www.ibge.gov.br/estatisticas/sociais/pop ulacao/9109-projecao-da-populacao.html?edicao $=21830 \& \mathrm{t}=$ resultados.

Manzini, E. J. (2015). Entrevista semiestruturada: análises de objetos e de roteiros. Departamento de Educação Especial, Programa de Pós-Graduação em Educação, Universidade Estadual Paulista. Disponível: https://wp.ufpel.edu.br/consagro/files/2012/03/MANZINI-Jos\%C3\%A9-Eduardo-Entevista-semiestruturada-An\%C3\%A1lise-de-objetivos-e-de-roteiros.pdf.

Martins, E. R. C. et al. (2020a). Young men's health from the perspective of health promotion and disease prevention. Brazilian Journal of Health. 3(2), 23012308. 10.34119/bjhrv3n2-081

Martins, E. R. C. et al. (2020b). Young men's health from the perspective of health promotion and disease prevention. Research, Society and Development, 9(9), e400997351. 10.33448/rsd-v9i9.7351 
Research, Society and Development, v. 10, n. 10, e566101019171, 2021

(CC BY 4.0) | ISSN 2525-3409 | DOI: http://dx.doi.org/10.33448/rsd-v10i10.19171

Minayo, M. C. S. (2013). O desafio do conhecimento: pesquisa qualitativa em saúde. Hucitec.

Moreira, R. L. S. F., Fontes, W. D., \& Barbosa, T. M. (2014). Difficulties of the man in primary healthcare: the speech of nurses. Rev. Enferm. Ana Nery, 18(4), 615-621. https://doi.org/10.5935/1414-8145.20140087.

OPAS. Doenças cardiovasculares. Brasília, 2017. Disponível: https://www.paho.org/bra/index.php?option=com_content\&view=article\&id=5253:doencascardiovasculares\&Itemid $=839$.

Pozzati, R. et al. (2013). Health care in men: reality and perspectives. Rev. Enfermagem UERJ, 21(4), 540-545. https://www.epublicacoes.uerj.br/index.php/enfermagemuerj/article/view/10032.

Silva, P. N. (2015). Gênero, masculinidade e saúde do homem: a representação social do agente comunitário de saúde. Dissertação de mestrado, Centro de Pesquisas René Rachou, Fundação Oswaldo Cruz. Belo Horizonte, MG, Brasil. https://www.arca.fiocruz.br/bitstream/icict/10738/2/Diss erta\%c3\%a7\%c3\%a3o_CHSS_PriscilaNevesSilva.pdf.

Stevens, B. et al. (2018). The Economic Burden of Heart Conditions in Brazil. Arq. Bras. Cardiol. 111(1), 29-36. http://www.dx.doi.org/10.5935/abc.20180104

Teixeira, D. B. S., \& Cruz, S. P. L. (2016). Health care for the human: analysis of its strength is search for the health services. Revista Cubana de Enfermería. 32(4). http://www.revenfermeria.sld.cu/index.php/enf/article/view/985/209.

Zibetti, M. L. T., \& Pereira, S. R. (2010). Women and teachers: repercussions of double duty on life conditions and on teaching work. Educar em revista, esp. 2, 259-276, https://www.scielo.br/scielo.php?script=sci_arttext\&pid=S0104-40602010000500016. 